SMALL INTESTINE

\title{
18F-fluoro-deoxy-glucose positron emission tomography (18F-FDG-PET) for assessment of enteropathy-type T cell lymphoma
}

\author{
M Hoffmann, H Vogelsang, K Kletter, G Zettinig, A Chott, M Raderer
}

Gut 2003;52:347-351

See end of article for authors' affiliations

Correspondence to: DrM Hoffmann,

Department of Nuclear Medicine, University of Vienna, AKH, Waehringer Guertel 18-20, A-1090 Vienna, Austria: martha.hoffmann@akhwien.ac.at

Accepted for publication 17 September 2002

\begin{abstract}
Background and aims: Enteropathy-type T cell lymphoma (ETCL) represents a relatively rare disease, accounting for less than $1 \%$ of non-Hodgkin's lymphomas. ETCL is an aggressive lymphoma which may either present de novo or arise in the context of longstanding or untreated coeliac disease (CD). The aim of this study was to evaluate the potential of 18F-fluoro-deoxy-glucose positron emission tomography (18F-FDG-PET) for imaging of ETCL. Furthermore, we wished to evaluate whether the presence of $C D$ might provide a potential diagnostic obstacle to imaging of lymphoma due to unspecific $18 \mathrm{~F}$ FDG uptake and whether accumulation of $18 \mathrm{~F}-\mathrm{FDG}$ within the gut correlates with activity of $C D$.

Patients and methods: We retrospectively analysed patients with ETCL and individuals suffering from CD undergoing 18F-FDG-imaging at our PET unit. Material for histological reassessment by a reference pathologist had to be available for inclusion of patients in the analysis. Whole body 18F-FDG-PET scans were performed 40 minutes following injection of 300-380 MBq of 18F-FDG. Images were reconstructed iteratively. In areas with focally elevated FDG uptake and in case of diffusely elevated intestinal 18F-FDG accumulation, standard uptake values (SUVs) were calculated. Results: During a period of two years, five patients (one male, four female) with a mean age of 56.4 years (range 44-62) with a diagnosis of ETCL underwent 18F-FDG-PET. Four of these patients were imaged before application of cytotoxic treatment while one patient had regular PET scans for follow up. All four patients undergoing pre-therapeutic imaging showed markedly elevated intestinal 18F-FDG uptake, with a maximal SUV of 6.4-8.0 (mean 7.15 (SD 0.82)). The patient imaged following surgery and cytotoxic therapy had no pathologic 18F-FDG uptake which was found to correlate with normal duodenal mucosa, as evidenced by repeated biopsies and conventional imaging methods. During the same time span, 12 patients (five male, seven female) with a mean age of 63.8 years (range 42-82) suffering from CD were imaged. Four of these patients showed no elevated intestinal 18F-FDG uptake while five had minor diffuse intestinal 18F-FDG accumulation with SUVs ranging between 2.2 and 4.6 (mean 3.4 (SD 0.89)). In the remaining three patients with diffuse intestinal 18F-FDG uptake, no SUV could be calculated. SUVs in patients with ETCL were remarkably higher than in patients suffering from $C D(p=0.011)$, irrespective of the activity of $C D$ at the time of imaging.

Conclusion: In spite of the relatively small number of patients, our results clearly indicate the potential value of 18F-FDG-PET for diagnosing and imaging ETCL. In addition, the data also suggest that $18 \mathrm{~F}$ FDG-PET may lead to early diagnosis in individuals developing ETCL in the context of longstanding CD. This is due to the fact that 18F-FDG does not appear to significantly accumulate in the gut of patients with $C D$, irrespective of disease activity.
\end{abstract}

$\mathrm{T}$ he gastrointestinal tract constitutes the most common site of origin for extranodal lymphomas. The large majority of these lymphomas are B cell derived ${ }^{1}$ but neoplasms with a putative $\mathrm{T}$ cell or natural killer cell origin are encountered. ${ }^{1-3}$ These lymphomas usually involve the upper small intestine and are very rare, accounting for less than $1 \%$ of all nonHodgkin's lymphomas and approximately 5\% of primary gastrointestinal lymphomas. ${ }^{45}$

An association between small intestinal lymphoma and villous atrophy of the mucosa not involving the lymphoma was first noted by Isaacson and Wright in 1978. ${ }^{6}$ Owing to the histological characteristics, they assumed that this condition might be a malignancy arising from histiocytes. A later report by Isaacson et al however, demonstrated monoclonal rearrangement of T cell receptor $\beta$ (TCR- $\beta$ ) and expression of T cell associated antigens consistent with a lymphoma of $\mathrm{T}$ cell origin. $^{7}$ In the recent WHO classification of tumours of haematopoeitic and lymphoid tissues, ${ }^{8}$ this type of lymphoma is included under the term "enteropathy-type T cell lymphoma" (ETCL). ${ }^{9}$
ETCL is an aggressive disease which may either present de novo or arise in the context of longstanding or untreated coeliac disease $(C D) .^{1-3}{ }^{10-13}$ The occurrence of refractory spruethat is, clinical and histological worsening of CD in spite of a gluten free diet-or the development of ulcerative jejunitis may herald the progression of CD to overt lymphoma, as monoclonal $\mathrm{T}$ cell populations have been demonstrated in refractory sprue, ulcerative jejunitis, and ETCL. ${ }^{11}{ }^{14}$

According to recent data, patients diagnosed with ETCL face a poor prognosis. ${ }^{315}$ This is largely due to complications arising from peritonitis and malnutrition and later from progressive disease typically characterised by intestinal recurrences. Therefore, only approximately $50 \%$ of patients are amenable to chemotherapy and only a small proportion can finish the

Abbreviations: $C D$, coeliac disease; $C T$, computed tomography; $E M A$, endomysial antibodies; ETCL, enteropathy-type T cell lymphoma; FDG, fluoro-deoxy-glucose; PET, positron emission tomography; ROI, region of interest; SUV, standard uptake value; TCR- $\beta$, T cell receptor $\beta$. 
treatment as scheduled. While occurrence of an aberrant phenotype of intraepithelial $\mathrm{T}$ cells and a monoclonal TCR rearrangement are suggestive of ETCL, ${ }^{23}{ }^{10}{ }^{11}$ the final diagnosis might be difficult to establish from duodenal biopsies. In addition, conventional radiological imaging appears to be of rather limited value in patients with ETCL as the neoplastic changes may be restricted to the epithelial layer of the small bowel even when the lymphoma diffusely affects the whole small intestine. Thus methods with the potential to delineate the transition from CD to overt lymphoma and to assess the tumour load in affected patients are strongly warranted to facilitate early diagnosis and avoid delayed institution of treatment.

Fluorine-18-2-fluoro-2-deoxy-D-glucose (18F-FDG) is one of the most widely applied positron emission tomography (PET) tracers used to survey cell metabolism. As the metabolic turnover of tumour cells usually exceeds physiological metabolic activity, excessive 18F-FDG uptake has consequently been demonstrated in most cancers in vivo, rendering whole body 18F-FDG-PET an excellent staging method in various malignancies. ${ }^{16-18}$ Especially in the field of malignant lymphomas, 18F-FDG-PET has become a well accepted method for diagnosis and staging. ${ }^{19}{ }^{20}$ One exception however appears to be extranodal marginal zone B cell lymphoma of the mucosa associated lymphoid tissue (MALT lymphoma), which cannot be imaged by means of 18F-FDG-PET. ${ }^{21}$ As $18 \mathrm{~F}-$ FDG-PET has been used successfully for imaging of aggressive lymphomas, it appears to be an attractive tool for clinical use in ETCL, as it can image the whole body, including the small intestine. Unspecific uptake of 18F-FDG however has also been reported in various inflammatory conditions, and one cannot rule out the fact that the inflammatory background in intestinal mucosa affected by CD may constitute a potential drawback for successful imaging of early stage ETCL.

The objective of this study therefore was to evaluate the potential of 18F-FDG-PET for imaging of ETCL. Apart from this proof-of-principle approach, we have compared the results obtained in ETCL with 18F-FDG-PET findings in individuals with $\mathrm{CD}$ in order to evaluate intestinal 18F-FDG uptake in this cohort of patients.

\section{PATIENTS AND METHODS}

A retrospective analysis of patients with ETCL and individuals suffering from CD undergoing imaging at our PET unit was performed. Data of patients identified from our archives were reassessed with respect to histological diagnosis and clinical data, including history of enteropathy, gluten free diet, presence of endomysial antibodies (EMAs), and availability of histological samples for re-evaluation. Histological diagnosis of ETCL was established by a reference pathologist (AC) according to the recent WHO Classification of Tumours of Haematopoietic and Lymphoid Tissues. ${ }^{9}$ Biopsies from patients with $\mathrm{CD}$ were graded according to the criteria established by Marsh and modified by Oberhuber and colleagues. ${ }^{22}$ Only patients with histological samples available at our institution were included in this analysis. EMAs were determined in serum by immune fluorescence microscopy on slides of distal monkey oesophagus (Bios, Barcelona, Spain) using rabbit anti- IgA. ${ }^{23}$

Whole body 18F-FDG-PET scans were performed on a GE Advance PET scanner (GE Medical Systems, Waukesha, Wisconsin, USA) with a whole body mode implemented as standard software. All patients were asked to fast for at least four hours prior to the 18F- FDG-PET scan. All patients received an intravenous injection of $20 \mathrm{mg} N$-butylbromide (Buscopan; Boehringer Ingelheim, Germany) for smooth muscle relaxation. Blood glucose levels were normal in all patients at the time of 18F-FDG application. PET emission scanning was started 40 minutes following an intravenous bolus injection of $300-380 \mathrm{MBq}$ of $18 \mathrm{~F}-\mathrm{FDG}$ and was performed from the neck to at least the pelvic floor. Emission scans of five minutes acquisition time and subsequent transmission scans of four minutes ( 75000 000 counts) per bed position were obtained. Transmission data were smoothed by a one dimensional filter in the axial direction and segmented, and attenuation correction factors were calculated by forward projection. Images were reconstructed iteratively. In areas with focally elevated 18F-FDG uptake and in case of diffusely elevated intestinal 18F-FDG accumulation, a standard uptake value (SUV) was calculated. SUV was determined according to the standard formula, as activity in the region of interest (ROI) given in becquerels (Bq)/ml/injected dose in $\mathrm{Bq} /$ per weight $(\mathrm{kg})$. ROIs were drawn manually around areas of diffuse or focal 18F-FDG uptake. In three patients with minor diffuse 18F-FDG uptake in the small bowel, no transmission scans were available, and thus no calculation of SUV could be performed. In the absence of pathological 18F-FDG uptake, no SUV was calculated.

18F-FDG-PET results were correlated with histological findings of duodenal biopsies and with conventional imaging methods (computed tomography (CT), enteroclysma CT) and surgical findings, respectively.

Statistical data are given as mean (SD) and the respective range. For statistical analysis the Mann-Whitney U test was used to compare SUV levels of patients suffering from ETCL and $C D$, respectively. A p value $\leqslant 0.05$ was considered statistically significant.

\section{RESULTS}

During a period of two years, five patients (four female, one male), mean age 56.4 years (range 44-62), diagnosed with ETCL were referred to our PET unit (table 1). Diagnosis had been established on duodenal biopsies in three patients and on surgical resection specimens in two patients (fig 1). Four of these patients had enteropathic changes in non- tumoral mucosa which were classified according to Oberhuber and colleagues $^{22}$ as type 1 (increased number of intraepithelial lymphocytes in an otherwise normal mucosa, $n=1)$, type $3 a$ (increased number of intraepithelial lymphocytes and mild villous atrophy, $\mathrm{n}=2$ ), and type $3 \mathrm{c}$ (increased number of intraepithelial lymphocytes and flat mucosa, $n=1$ ). In two of these patients the small bowel mucosa distant from the invasive ETCL showed a striking intraepithelial lymphocytosis suggestive of an intraepithelial "low grade" component. In one of these two cases immunophenotypic analysis and molecular studies indeed confirmed intraepithelial lymphomatosis by demonstrating the same aberrant immunophenotype and the same monoclonal TCR gamma chain rearrangement in sections from intraepithelial and invasive lymphoma. Only two of these patients were known to have a history of documented CD. Both patients were on a gluten free diet, and had shown clinical deterioration in spite of strict adherence to dietary measures before diagnosis of ETCL. One patient had an eight month history of intestinal ulceration before the diagnosis of $\mathrm{T}$ cell lymphoma involving the intestine and peripheral lymph nodes could be established.

During the same time span, 12 patients (seven female, five male) with a mean age of 63.8 years (range 42-82) with CD were imaged in our PET unit using identical examination algorithms. On imaging, four of these patients had clinical worsening as a result of non- compliance with a gluten free diet while three additional patients presented with refractory sprue-that is, worsening of symptoms in spite of adherence to a gluten free diet. In none of these patients were signs of overt lymphomatous transformations or criteria diagnostic for intraepithelial lymphoma found on histological assessment. Mean time span on a gluten free diet prior to PET scan was 67 months (range 4-212) but four patients were non-compliant at the time of the PET scan (table 2). 
Table 1 Characteristics of patients with enteropathy-type intestinal T cell lymphoma (ETCL)

\begin{tabular}{|c|c|c|c|c|c|}
\hline $\begin{array}{l}\text { Patient } \\
\text { No }\end{array}$ & Sex/Age & $\begin{array}{l}\text { Clinical signs of } \\
\text { entheropathy }\end{array}$ & Histology & PET result & suV \\
\hline 1 & $\mathrm{~F} / 60$ & $C D(20 *)$ & ETCL & Focal intestinal FDG uptake & 7.7 \\
\hline 2 & $\mathrm{~F} / 58$ & Intestinal ulceration & ETCL & Focal intestinal FDG uptake & 6.4 \\
\hline 3 & $M / 62$ & No clinical signs & ETCL & $\begin{array}{l}\text { Focal intestinal and intrapulmonary FDG uptake } \\
\text { (confirmed by autopsy) }\end{array}$ & 6.5 \\
\hline 4 & $F / 58$ & $C D\left(12^{*}\right)$ & ETCL (after chemotherapy) & $4 \times$ follow up PET, $4 \times$ negative & $\mathrm{Neg}$ \\
\hline 5 & $\mathrm{~F} / 44$ & No clinical signs & ETCL & $\begin{array}{l}\text { 1 st PET: focal intestinal FDG uptake; 2nd PET (after } \\
\text { chemotherapy): negative }\end{array}$ & $\begin{array}{l}\text { 1st PET: } 8.0 \\
\text { 2nd PET: neg }\end{array}$ \\
\hline
\end{tabular}

PET, positron emission tomograph; CD, coeliac disease; SUV, standard uptake value.

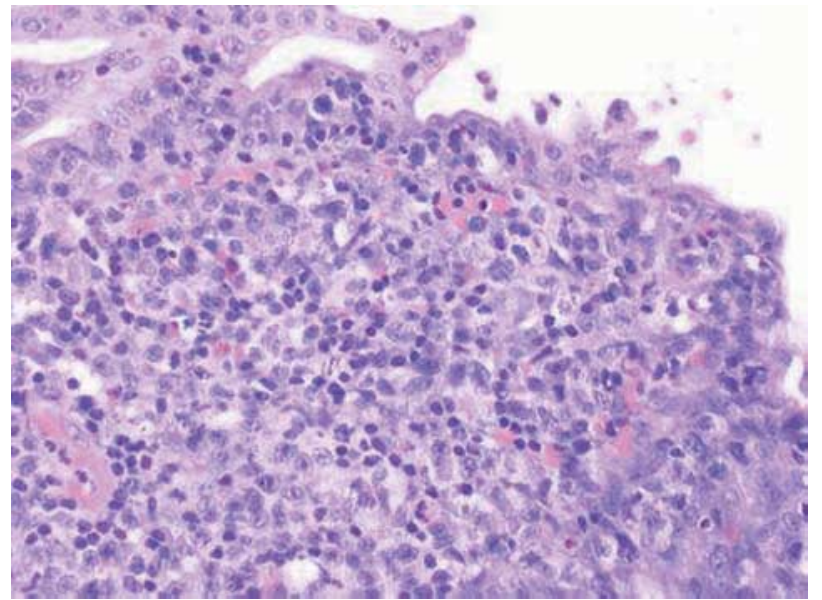

Figure 1 Jejunal infiltration by enteropathy-type T cell lymphoma (ETCL). Haematoxylin and eosin stained section showing jejunal mucosa largely replaced by ETCL composed of large atypical lymphoid cells.

In four ETCL patients the PET scan was performed prior to initiation of cytotoxic therapy. All of these patients showed significantly elevated abdominal focal or multifocal 18F-FDG uptake (fig 2A) at the site of bioptically verified lymphoma. In three cases, additional intestinal sites were also seen and one of these patients had an additional hot spot in the lung. While a CT scan of the thorax was suggestive of an inflammatory lesion, the PET result of lymphomatous involvement of both the intestine and lung was confirmed by autopsy four months later.
SUV in the cohort of patients with ETCL was $7.15(0.82)$ (range 6.4-8.0). In one of these patients a control 18F-FDGPET was acquired after six cycles of chemotherapy and disclosed no pathological 18F-FDG uptake. Currently, the patient is alive without evidence of disease 14 months after the initial diagnosis.

One of these patients was imaged following surgery and chemotherapy. Both 18F-FDG- PET and CT scanning were normal, and multiple duodenal biopsies and follow up CT scans obtained over a time span of 32 months showed no evidence of lymphoma recurrence. Thus the 18F-FDG-PET result was rated as true negative.

Of 12 patients with CD (for patient characteristics see table 2 ), four patients showed no elevated intestinal 18F-FDG uptake and thus no SUV was calculated in these patients. Five patients showed minor diffuse intestinal 18F-FDG accumulation with SUVs ranging from 2.2 to 4.6 (mean $3.4(0.89)$ ) (fig 2B). In an additional three patients with intestinal 18F-FDG uptake, no transmission scan had been obtained and thus SUV could not be calculated. SUVs in patients with ETCL were significantly higher than in patients suffering from $C D$ $(p=0.011)$. In patients with CD, intestinal 18F-FDG uptake did not correlate with disease activity (see table 2), as assessed according to the modified Marsh criteria. ${ }^{22}$ In four of 12 patients, a follow up 18F-FDG-PET was obtained. Three underwent imaging after initiation of dietary measures, resulting in clinical and histological improvement, while one patient was scanned due to clinical deterioration in spite of adherence to a gluten free diet (as also reflected by negative EMA levels). In spite of pronounced changes in disease status, 18F- FDG accumulation was not significantly altered in these four individuals. None of these patients with CD in our series

Table 2 Characteristics of patients with coeliac disease (CD)

\begin{tabular}{|c|c|c|c|c|c|c|c|}
\hline $\begin{array}{l}\text { Patient } \\
\text { No }\end{array}$ & Sex/Age & Diagn & Histo† & $\mathrm{EMA}^{*}$ & PET result & SUV & $\begin{array}{l}\text { Onset of gluten free die } \\
\text { prior to PET (months) }\end{array}$ \\
\hline 1 & $F / 58$ & $C D$ & 0 & 0 & Diffuse intestinal FDG uptake & 3.0 & 75 \\
\hline 2 & $\mathrm{~F} / 42$ & $C D$ & 0 & 0 & Negative & Negative & 35 \\
\hline 3 & $F / 59$ & $\mathrm{CD} \neq$ & $\begin{array}{l}3 c \\
0\end{array}$ & $\begin{array}{l}3+ \\
0\end{array}$ & Negative negative & Negative negative & $\begin{array}{l}212 \ddagger \\
223\end{array}$ \\
\hline 4 & $\mathrm{~F} / 74$ & RS & $\begin{array}{l}3 a \\
2 a\end{array}$ & $\begin{array}{l}1 \\
0\end{array}$ & Diffuse intestinal FDG uptake & $\begin{array}{l}\text { 1st PET: } 2.9 \\
\text { 2nd PET } 2.2\end{array}$ & $\begin{array}{l}140 \\
154\end{array}$ \\
\hline 5 & $F / 76$ & $\mathrm{CD} \neq$ & $\begin{array}{l}3 a \\
0\end{array}$ & $\begin{array}{l}3+ \\
0\end{array}$ & $\begin{array}{l}\text { Both PET scans: diffuse intestinal FDG } \\
\text { uptake }\end{array}$ & $\begin{array}{l}\text { 1 st PET: } 4.2 ; 2 \text { nd PET: no SUV } \\
\text { available }\end{array}$ & $\begin{array}{l}170 \neq \\
173\end{array}$ \\
\hline 6 & $M / 55$ & $C D$ & $3 c$ & 0 & Diffuse intestinal FDG uptake & 4.6 & 4 \\
\hline 7 & $M / 56$ & RS & $3 c$ & 0 & Intestinal FDG uptake & 3.5 & 60 \\
\hline 8 & $M / 58$ & $C D$ & $3 b-c$ & 1 & Negative & Negative & 4 \\
\hline 9 & $M / 63$ & $\mathrm{CD} \ddagger$ & $3 b$ & 3 & Minimal diffuse intestinal FDG uptake & No SUV available & $74 \ddagger$ \\
\hline 10 & $\mathrm{~F} / 76$ & $C D$ & $3 a$ & 0 & Negative & Negative & 72 \\
\hline 11 & $\mathrm{~F} / 67$ & $\mathrm{CD} \ddagger$ & $3 b$ & $3+$ & Minimal diffuse intestinal FDG uptake & No SUV available & 48 \\
\hline 12 & $M / 82$ & RS & $\begin{array}{l}3 a \\
3 b\end{array}$ & $\begin{array}{l}0 \\
0\end{array}$ & $\begin{array}{l}\text { 1st and 2nd PET: minimal diffuse } \\
\text { intestinal FDG uptake without dynamics }\end{array}$ & No SUV available & $\begin{array}{l}12 \\
32\end{array}$ \\
\hline
\end{tabular}

RS, refractory sprue; SUV, standard uptake value; FDG, fluoro-deoxy-glucose; PET, positron emission tomography

*EMA, endomysial antibodies semiquantitatively classified from 0 to 3.

tHistology according to modified Marsh criteria.

$\ddagger$ Non-compliant. 

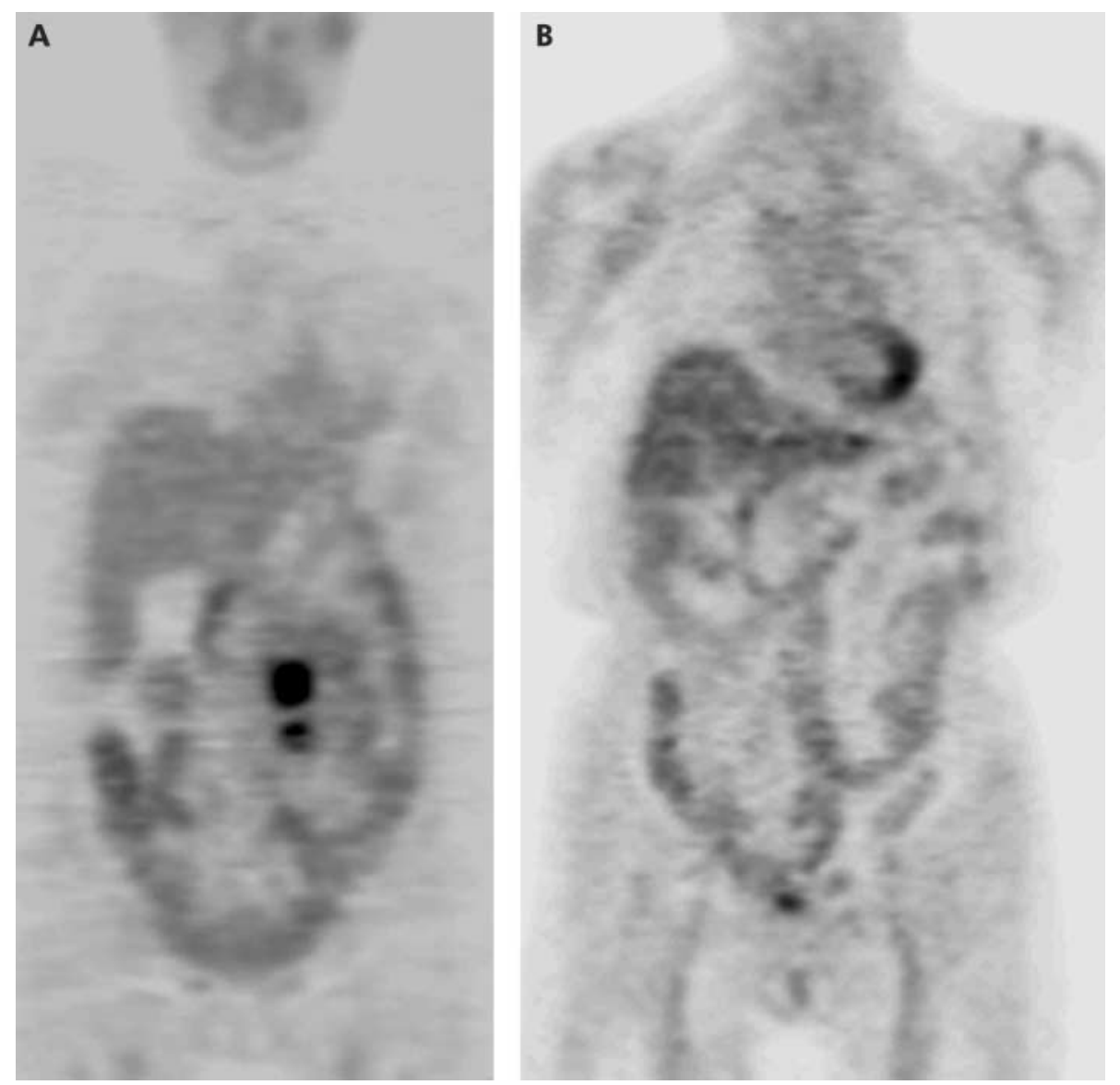

Figure 2 (A) Coronal 18F-fluoro-deoxy-glucose positron emission tomography (18F-FDG-PET) image of a 60 year old female patient suffering from enteropathy-type T cell lymphoma (ETCL) showing two lesions with elevated FDG uptake (standard uptake value (SUV) 7.7). (B) Coronal 18F-FDG-PET image of a 74 year old female patient suffering from refractory sprue showing diffuse intestinal FDG uptake (SUV 2.2).

developed ETCL within the mean observation period of 22 months (range 4-32 months after the first 18F-FDG-PET scan).

\section{DISCUSSION}

ETCL is a rare disease,${ }^{45}$ characterised by a dismal prognosis due to delayed diagnosis in most patients. The impaired performance status in afflicted individuals usually leads to poor tolerance of aggressive cytotoxic treatment, rendering current therapeutic approaches ineffective. ${ }^{15}$ While correct histological diagnosis is usually not a problem on resection specimens obtained from patients undergoing surgery because of intestinal perforation, it might be more difficult to establish on duodenal biopsies in patients with longstanding CD.

PET using 18F-FDG has become an established standard imaging modality in patients with lymphoma. ${ }^{20}{ }^{21}$ We therefore hypothesised that 18F-FDG-PET might also be a potential diagnostic tool in patients with ETCL. 18F-FDG however accumulates in rapidly proliferating tissues as a reflection of enhanced glucose metabolism in cells and is therefore not specific for tumour cells. Various benign processes have been reported to result in 18F-FDG uptake, including inflammation or even muscle contractions. ${ }^{24-26}$ In these cases, calculation of SUV usually helps to distinguish neoplastic from benign processes. ${ }^{27-29}$ The objective of our analysis was therefore to evaluate the efficacy of 18F-FDG-PET for imaging of ETCL with special emphasis on its potential enteropathic background caused by CD.

Our results demonstrated that 18F-FDG-PET can correctly visualise sites affected by ETCL. In addition, the 18F-FDG-PET results in patients with ETCL differed significantly from those obtained in patients suffering from CD. Visual analysis and particularly calculation of SUV revealed enhanced 18F-FDG uptake in involved intestinal areas, as confirmed on biopsy. In three patients, additional intestinal sites were found, and in one of these patients 18F-FDG-PET disclosed additional pulmonary involvement not evident before scanning. In terms of SUV, values were significantly higher in patients with ETCL compared with those with $\mathrm{CD}(\mathrm{p}=0.011)$. Eight of our patients with CD showed tracer uptake but none had an SUV higher than 5, while all patients with ETCL demonstrating enhanced 18F-FDG accumulation had an SUV $>6$. In patients with a normal gut mucosa-that is, absence of malignancy or inflammation-usually no accumulation of 18F-FDG within the gut was seen. However, in some patients smooth muscle contractions can result in diffuse intestinal 18F- FDG uptake. To exclude this, all of our patients received $N$-butylbromide for smooth muscle relaxation.

Interestingly, there was no distinctive PET feature to differentiate active from inactive CD. In our patients, accumulation of 18F-FDG within the intestinal tract did not correlate with overall disease activity, and did not significantly change in patients imaged at different time points, in spite of altered clinical symptoms and histological findings. Rather than resulting in a gradual increase in 18F-FDG uptake from inactive to active $\mathrm{CD}$ and refractory sprue, our results suggest that 18F-FDG-PET draws a precise threshold between CD and ETCL in terms of SUVs. As ETCL is a rare condition, the patient number in our retrospective study was rather small. While our limited data do not allow exact conclusions on whether or not 18F-FDG-PET is helpful in the follow up of patients with CD in diagnosing ETCL prior to other methods (biopsy, conventional 
imaging), our data suggest that individuals with an SUV $>6$ should be investigated further for ETCL. More prospective studies are needed to define the value of 18F-FDG-PET as a diagnostic tool in patients suffering from longstanding CD with suspected ETCL.

\section{Authors' affiliations}

M Hoffmann, K Kletter, G Zettinig, Department of Nuclear Medicine, Internal Medicine IV, University of Vienna, Vienna, Austria $\mathbf{H}$ Vogelsang, Division of Gastroenterology, University of Vienna, Vienna, Austria

A Chott, Division of Pathology, University of Vienna, Vienna, Austria M Raderer, Internal Medicine I, Division of Oncology, University of Vienna, Vienna, Austria

\section{REFERENCES}

1 Isaacson PG. Gastrointestinal lymphomas of T- and B-cell types. Mod Pathol 1999:12:151-8.

2 Chott A, Vesely M, Simonitsch I, et al. Classification of intestinal T-cell neoplasms and their differential diagnosis. Am J Clin Pathol 1999;111(suppl 1):S68-74.

3 Chott A, Haedicke W, Mosberger I, et al. Most CD56+ intestinal lymphomas are CD8+ CD5- T-cell lymphomas of monomorphic small to medium size histology. Am J Pathol 1998;153:1483-90.

4 International Lymphoma Study Group. The Non-Hodgkin's Lymphoma Classification Project: A clinical evaluation of the International Lymphoma Study Group classification of non-Hodgkin's lymphoma. Blood 1997;89:3909-18.

5 Chott A, Dragosics B, Radaszkiewicz T. Peripheral T-cell lymphomas of the intestine. Am J Pathol 1992;141:1361-71.

6 Isaacson PG, Wright DH. Intestinal lymphoma associated with malabsorption. Lancet 1978;2:67-70.

7 Isaacson PG, O'Connor N, Spencer J, et al. Malignant histiocytosis of the intestine: a T-cell lymphoma. Lancet 1985;2:688-91

8 Jaffe ES, Harris NL, Stein H, et al. World Health Organization classification of tumours: tumours of hematopoietic and lymphoid tissues. Lyon: IARC Press, 2001.

9 Isaacson PG, Wright D, Ralfkiaer $\mathrm{E}$, et al. Enteropathy-type T-cell lymphoma. In: Jaffe ES, Harris NL, Stein $\mathrm{H}$, et al, eds. World Health Organization classification of tumours: tumours of hematopoietic and lymphoid tissues. Lyon: IARC Press, 2001:208-9.

10 Isaacson PG. Gastrointestinal lymphoma. Hum Pathol 1994;25:1020-9

11 Daum S, Weiss D, Hummel $M$, et al. Frequency of clonal intraepithelial lymphocyte proliferations in enteropathy-type intestinal T cell lymphoma, coeliac disease, and refractory sprue. Gut 2001;49:804-12.
12 Holmes G, Stokes PL, Sorahan TM, et al. Coeliac disease, gluten-free diet, and malignancy. Gut 1976;17:612-19.

13 Holmes G, Prior P, Lane M, et al. Malignancy in coeliac disease-effect of gluten free diet. Gut 1989;30:333-8.

14 Bagdi E, Diss T, Munson P, et al. Mucosal intra-epithelial lymphocytes in enteropathy-associated T-cell lymphoma, ulcerative jejunitis and refractory celiac disease constitute a neoplastic population. Blood 1999;94:260-4.

15 Gale J, Simmonds P, Mead G, et al. Enteropathy-type intestinal T-cell lymphoma: Clinical features and treatment of 31 patients in a single center. J Clin Oncol 2000;18:795-803.

16 Rigo P, Paulus P, Kaschten BJ, et al. Oncological applications of positron emission tomography with fluorine- 18 fluorodesoxyglucose. Eur J Nucl Med 1996;23:1641-74.

17 Bomanii JB, Costa DC, Ell PJ. Clinical role of positron emission tomography in oncology. Lancet Oncol 2001;2:157-64.

18 Czernin J, Phelps ME. Positron emission tomography scanning: current and future applications. Annu Rev Med 2002;53:89-1 12.

19 Schoder H, Meta J, Yap C, et al. Effect of whole-body (18)F-FDG PET imaging on clinical staging and management of patients with malignant lymphoma. J Nucl Med 2001;42:1 139-43.

20 Segall GM. FDG PET imaging in patients with lymphoma: a clinical perspective. Nucl Med 2001;42:609-10.

21 Hoffmann $M$, Kletter $K$, Diemling $M$, et al. Positron emission tomography with fluorine-18-2-fluoro-2-deoxy-D-glucose (F18-FDG) does not visualize extranodal B-cell lymphoma of the mucosa-associated lymphoid tissue (MALT)-type. Ann Oncol 1999;10:1 185-9.

22 Oberhuber G, Granditsch G, Vogelsang H. The histopathology of coeliac disease: time for standardized report scheme for pathologists. Eur J Gastroenterol Hepatol 1999;11:1 185-94.

23 Ferreira $M$, Davies SL, Butler $M$, et al. Endomysial antibody: is it the best screening test for coeliac disease? Gut 1992;33:1633-7.

24 Zhuang $\mathbf{H}$, Pourdehnad $M$, Lambright ES, et al. Dual time point 18F-FDG PET imaging for differentiating malignant from inflammatory processes. J Nucl Med 2001;42:1412-17.

25 Bakheet SM, Powe J. Benign causes of 18-FDG uptake on whole body imaging. Semin Nucl Med 1998;28:352-8.

26 Sugawara Y, Braun DK, Kison PV, et al.I. Rapid detection of human infections with fluorine- 18 fluorodeoxyglucose and positron emission tomography: preliminary results. Eur J Nucl Med 1998;25: 1238-43.

27 Aoki J, Watanabe H, Shinozaki T, et al. FDG PET of primary benign and malignant bone tumours: standardized uptake value in 52 lesions. Radiology 2001;219:774-7.

28 Huang SC. Anatomy of SUV. Standardized uptake value. Nucl Med Biol 2000;27:643-6.

29 Lowe VJ, Fletcher JW, Gobar L, et al.I. Prospective investigation of positron emission tomography in lung nodules. J Clin Oncol $1998 ; 16: 1075-84$ 